\title{
Application of the Direct Distance Estimation procedure to eclipsing binaries in star clusters
}

\author{
E. F. Milone ${ }^{1}$ and S. J. Schiller ${ }^{2}$ \\ ${ }^{1}$ Rothney Astrophysical Observatory, University of Calgary, 2500 University Dr., NW, \\ Calgary, AB T2N 1N4, Canada \\ email: milone@ucalgary.ca \\ ${ }^{2}$ Raytheon Space and Airborne Systems, 2000 E. El Segundo Blvd, M/S EO/E04/N187, \\ El Segundo, CA 90245, USA \\ email: stephen_j_schiller@raytheon.com
}

\begin{abstract}
We alert the community to a paradigm method to calibrate a range of standard candles by means of well-calibrated photometry of eclipsing binaries in star clusters. In particular, we re-examine systems studied as part of our Binaries-in-Clusters program, and previously analyzed with earlier versions of the Wilson-Devinney light-curve modeling program. We make use of the 2010 version of this program, which incorporates a procedure to estimate the distance to an eclipsing system directly, as a system parameter, and is thus dependent on the data and analysis model alone. As such, the derived distance is accorded a standard error, independent of any additional assumptions or approximations that such analyses conventionally require.
\end{abstract}

Keywords. binaries: eclipsing, stars: distances, stars: individual (HD 27130, DS Andromedae), open clusters and associations: general, open clusters and associations: individual (Hyades, NGC $752)$

\section{Introduction}

The use of eclipsing binaries to determine distances to the stellar ensembles in which they are embedded is well-established: see, for example, Kallrath \& Milone (2009), which also discusses the Direct Distance Estimation (DDE) procedure and its limitations. As part of a long-standing program to study binaries in star clusters, we previously analyzed several open-cluster eclipsing systems with earlier versions of the Wilson-Devinney (WD) light-curve modeling program. The current work is carried out with the 2010 version of WD (see Wilson 2008; Wilson et al. 2010). This incorporates the DDE procedure, by which the distance is determined as a system parameter, thus avoiding post-analysis inconsistencies, such as having to assume stellar sphericity to compute the luminosity. DDE also provides a well-determined mean standard error for the resulting distance. Consequently, the method provides a powerful way to calibrate the distances to other objects in the cluster in which the analyzed eclipsing binaries are found, with the added (although usually small) uncertainty of the relative distances of the other objects within the cluster and the analyzed targets. Previous applications of DDE to eclipsing binaries in the field can be found in

(a) Wilson \& Van Hamme (2009): RS Cha, WW Aur, R CMa, RZ Cnc, RZ Cas, and AW UMa;

(b) Wilson et al. (2010): TZ Men, V1130 Tau, TY Pyx, V505 Per, $\varepsilon$ CrA, BG Ind, and WW Aur; and

(c) Wilson \& Raichur (2012): V1143 Cyg, $\varepsilon$ CrA, and ER Vul. 
The current study is the first application of the algorithm to an eclipsing binary in a star cluster.

\section{Current analysis}

We use as starting parameters those obtained in fully convergent solutions (see Schiller \& Milone 1987, 1988; Milone et al. 1995 - for work on HD 27130 in the Hyades, and DS And and Heinemann $235=$ QX And in NGC 752). Here we report preliminary results only for the the double-lined eclipsing binary in the Hyades, HD $27130=$ van Bueren $22=$ V818 Tau. The system offers several advantages for such a study:

(a) HD 27130 is a confirmed Hyades member and the nearest eclipsing system in a cluster;

(b) both primary and secondary minima are observed;

(c) there are calibrated five-passband photometric data (Schiller \& Milone 1987); and

(d) there are excellent-quality radial-velocity (RV) data (McClure 1982; Griffin et al. 1985).

There are two impediments to high-precision parameter determination, however. The secondary eclipses in this partially eclipsing system are very shallow, especially in the $U$ and $B$ passbands, and there is significant extra-eclipse variation in the system, attributable to active regions on at least one of the two components. The latter is most clearly demonstrated in the primary, hotter component, with a migrating wave of variable amplitude $(\leqslant 3 \%$ in $V)$, and strong correlation between $V$-band magnitude and $(B-V)$ color index. Because of this variation, Schiller \& Milone (1987) normalized the minima to the adjoining maxima on a cycle-by-cycle basis. In addition to dark star-spot effects, one flare was detected in the photometry, so the light curves exhibit both bright and faint extra-eclipse excursions. In the current analysis, the standardized magnitudes, variations and all, were analyzed. The data were analyzed in suites of RVs plus two passbands, as prescribed by Wilson (2008). The fitted RV and light curves for the RV $+B V$ data suite are shown in Fig. 1.

Absolute parameters of the converged model fits to each of the data sets are listed in Table 1 .

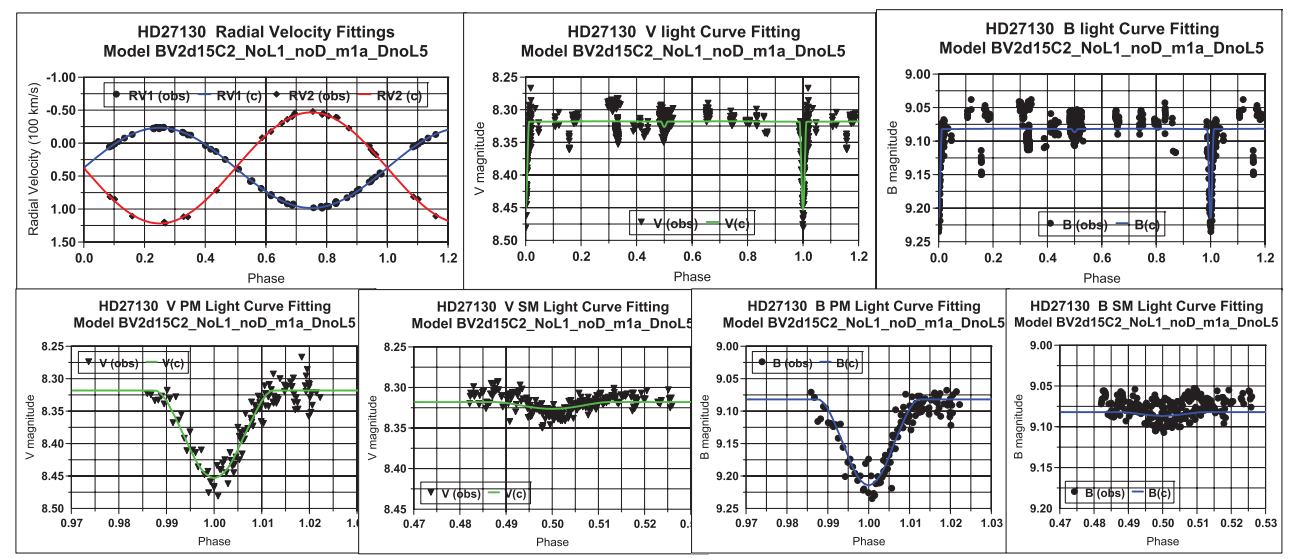

Figure 1. Radial-velocity and light curves of the RV $+B V$ suite of data for the Hyades doublelined eclipsing-binary system HD $27130=$ V818 Tau, fitted to a converged model with $[\mathrm{m} / \mathrm{H}]=$ 0.100 dex and $T_{1}=5470 \mathrm{~K}$. 
Table 1. Absolute parameters from analyses of three data sets of HD $27130 .^{1}$

\begin{tabular}{lccc}
\hline Parameters & $\mathrm{RV}+V I_{\mathrm{C}}$ & $\mathrm{RV}+B V$ & $\mathrm{RV}+B I_{\mathrm{C}}$ \\
\hline$M_{1}\left(\mathrm{M}_{\odot}\right)$ & 1.0422 & 1.0398 & 1.0401 \\
$M_{2}\left(\mathrm{M}_{\odot}\right)$ & 0.7512 & 0.7507 & 0.7501 \\
$R_{1}\left(\mathrm{R}_{\odot}\right)$ & 0.9975 & 1.0256 & 0.9804 \\
$R_{2}\left(\mathrm{R}_{\odot}\right)$ & 0.7027 & 0.6875 & 0.6255 \\
$T_{1}{ }^{2}(\mathrm{~K})$ & 5470 & 5470 & 5470 \\
$T_{2}(\mathrm{~K})$ & 3854 & 3655 & 3884 \\
$M_{\mathrm{bol}, 1}(\mathrm{mag})$ & 4.99 & 4.93 & 5.03 \\
$M_{\mathrm{bol}, 2}(\mathrm{mag})$ & 7.27 & 7.55 & 7.49 \\
$\log g_{1}(\mathrm{cgs})$ & 4.46 & 4.43 & 4.47 \\
$\log g_{2}(\mathrm{cgs})$ & 4.62 & 4.64 & 4.72 \\
$d(\mathrm{pc})^{3}$ & $44.77(52)$ & $45.50(105)$ & $43.70(52)$ \\
$(m-M)(\mathrm{mag})$ & $3.255(25)$ & $3.290(50)$ & $3.203(26)$ \\
\hline
\end{tabular}

Notes:

${ }^{1}$ For this model we assume $[\mathrm{m} / \mathrm{H}]=0.100$ dex, close to the metallicity determined by Taylor \& Joner (2005);

${ }_{2}^{2}$ Assumed and unadjusted temperature of the hotter component;

${ }^{3}$ Quantities in parentheses following the distances and distance moduli in the last two rows are the mean standard errors in units of the last decimal place.

The model applied to the data sets of Table 1 assumes a metallicity $[\mathrm{m} / \mathrm{H}]=0.100$ dex, a value for which discrete atmospheric-flux grid tables are available. This metallicity is close to the 0.103 dex value determined by Taylor \& Joner (2005). Two other models were also studied.

\section{Results and Conclusion}

The weighted mean of the distance values in Table 1 is $1.647(3)$ for $\log d$, or $44.37(34)$ pc for $d$, where the uncertainties in parentheses are given in units of the last decimal place. Solar-composition models for the same three data suites in Table 1 yield $\log d$ values of $1.647(6), 1.648(9)$, and 1.636(5) in the same order, corresponding to distances of 44.36(61), 44.46(92), and 43.25(50) pc, respectively, for weighted means of $\log d=$ $1.642(3)$, corresponding to $d=43.81(33) \mathrm{pc}$. We have also explored the effect on the distance of increasing the assumed temperature of the hotter component to $5570 \mathrm{~K}$, as suggested by Schiller \& Milone (1987), as a better match to the $(B-V)$ color index. For the higher- $T_{1},[\mathrm{~m} / \mathrm{H}]=0.100$ dex model, we find that the same three data suites yield $\log$ $d=1.624(3), 1.633(6)$, and 1.639(6), corresponding to distances of 42.07(29), 42.95(59), and $43.57(63)$. The weighted means of this higher- $T_{1}$ model are $\log d=1.628(3)$ and $d=$ $42.44(29)$ pc. For a given model, it can be seen that the data suites produce distances that are not significantly different, within the errors. The solar and nonsolar means agree within the errors, whereas the mean of models with the hotter component having a higher temperature by $100 \mathrm{~K}$ produces a significantly different result, especially if only the $B V$ and $V I$ suites are considered. Thus, for the two low- $T_{1}$ models, the DDE procedure seems to yield robust determinations of the distance, despite the clear evidence of stellar activity in these components. The broad scatter in the maxima permits determination of a mean light level, but at the cost of less precise parameters and possible systematic error. In any case, the weighted averages of all three model means are $\log d=1.628(3)$, $d=42.44(41) \mathrm{pc}$, and $(m-M)=3.139(21) \mathrm{mag}$, not significantly different from the distance derived from Hipparcos trigonometric-parallax measurements, $d=45.79_{-2.25}^{+2.50}$ pc (van Leeuwen 2007). In the absence of systematic errors in any of these distances, HD 
27130 is slightly closer than the cluster center, which (based on Hipparcos kinematic data of selected likely members) is $46.34(27)$ pc (Perryman et al. 1998), a result essentially confirmed by the more recent Hipparcos investigation of van Leeuwen (2009), who found a distance of $46.45(50) \mathrm{pc}$, with a somewhat different selection of probable members.

Work continues on this system to explore models in which both $T_{1}$ and $T_{2}$ are adjusted, with full treatment of migrating active regions, and on the DS Andromedae (And) and QX And systems in the intermediate-age open cluster NGC 752.

\section{Acknowledgements}

Our earlier work on these eclipsing systems and clusters was supported by NSERC of Canada. EFM thanks the IAU for a travel grant to attend the General Assembly and Symposium 289. It is a pleasure also to thank Robert E. Wilson for his generous help with our operation of WD 2010.

\section{References}

Griffin, R. E., Gunn, J. E., Zimmerman, B. A., \& Griffin, R. E. M. 1985, AJ, 90, 609

Kallrath, J., \& Milone, E. F. 2009, Eclipsing Binary Stars: Modeling and Analysis, $2^{\text {nd }}$ ed. (Springer Science+Business Media, Astronomy \& Astrophysics Library), pp. 27-28, 231ff McClure, R. D. 1982, ApJ, 254, 606

Milone, E. F., Stagg, C. R., Sugars, B. J. A. McVean, J. R., Schiller, S. J., Kallrath, J., \& Bradstreet, D. H. 1995, AJ, 109, 359

Perryman, M. A. C., Brown, A. G. A., Lebreton, Y., et al. 1998, A\&A, 331, 81

Schiller, S. J., \& Milone, E. F. 1987, AJ, 93, 1471

Schiller, S. J., \& Milone, E. F. 1988, AJ, 95, 1466

Taylor, B. J. \& Joner, M. D. 2005, ApJS, 159, 100

van Leeuwen, F. 2007, $A \mathscr{E} A, 474,653$

van Leeuwen, F. 2009, $A \mathscr{E} A$, 497, 209

Wilson, R. E. 2008, ApJ, 647, 676

Wilson, R. E. \& Raichur, H. 2012, MNRAS, 415, 596

Wilson, R. E. \& Van Hamme, W. 2009, ApJ, 699, 118

Wilson, R. E., Van Hamme, W., \& Terrell, D. 2010, ApJ, 723, 1469 